\title{
Walking speed-related changes in stride time variability: effects of decreased speed
}

\author{
Olivier Beauchet ${ }^{*+1}$, Cedric Annweiler ${ }^{1}$, Yhann Lecordroch ${ }^{2}$, Gilles Allali ${ }^{3}$, \\ Veronique Dubost ${ }^{4}$, François R Herrmann ${ }^{2}$ and Reto W Kressig ${ }^{\dagger 5}$
}

\begin{abstract}
Address: ${ }^{1}$ Department of Internal Medicine and Geriatrics, Angers University Hospital, UPRES UNAM EA 2646, University of Angers, UNAM, France, ${ }^{2}$ Department of Rehabilitation \& Geriatrics, Geneva University Hospitals, Switzerland, ${ }^{3}$ Department of Neurology, Geneva University Hospitals, Switzerland, ${ }^{4}$ FORMADEP, Korian, France and ${ }^{5}$ Department of Geriatrics, Basel University Hospital, Switzerland

Email: Olivier Beauchet* - olbeauchet@chu-angers.fr; Cedric Annweiler - cedric.annweiler@hotmail.fr;

Yhann Lecordroch - Yhann.Lecordroch@hcuge.ch; Gilles Allali - gilles.allali@hcuge.ch; Veronique Dubost - v.dubost@groupe-korian.com; François R Herrmann - francois.herrmann@hcuge.ch; Reto W Kressig - RKressig@uhbs.ch

* Corresponding author †Equal contributors
\end{abstract}

Published: 5 August 2009

Journal of NeuroEngineering and Rehabilitation 2009, 6:32 doi:10.1 186/1743-0003-6-32

This article is available from: http://www.jneuroengrehab.com/content/6/I/32

(c) 2009 Beauchet et al; licensee BioMed Central Ltd.

This is an Open Access article distributed under the terms of the Creative Commons Attribution License (http://creativecommons.org/licenses/by/2.0), which permits unrestricted use, distribution, and reproduction in any medium, provided the original work is properly cited.
Received: 26 August 2008

Accepted: 5 August 2009

\begin{abstract}
Background: Conflicting results have been reported regarding the relationship between stride time variability (STV) and walking speed. While some studies failed to establish any relationship, others reported either a linear or a non-linear relationship. We therefore sought to determine the extent to which decrease in self-selected walking speed influenced STV among healthy young adults.

Methods: The mean value, the standard deviation and the coefficient of variation of stride time, as well as the mean value of stride velocity were recorded while steady-state walking using the GAITRite ${ }^{\circledR}$ system in 29 healthy young adults who walked consecutively at $88 \%, 79 \%, 71 \%, 64 \%$, $58 \%, 53 \%, 46 \%$ and $39 \%$ of their preferred walking speed.

Results: The decrease in stride velocity increased significantly mean values, SD and CoV of stride time $(p<0.00 \mathrm{I})$, whereas the repetition of trials $(p=0.534, p=0.177$ and $p=0.69 \mathrm{I}$ respectively for mean, SD, CoV); and step asymmetry $(p=0.97 \mathrm{I}, p=0.150$ and $p=0.288$ for mean, SD and CoV) had no significant effect. Additionally, the subject's effect was significant for all stride parameters $(p<0.001)$. The relationship between a decrease in walking speed and all stride parameters (i.e., mean values, SD and CoV of stride time) was significantly quadratic and showed higher STV at a slow speed $(p<0.001)$.

Conclusion: The results support the assumption that gait variability increases while walking speed decreases and, thus, gait might be more unstable when healthy subjects walk slower compared with their preferred walking speed. Furthermore, these results highlight that a decrease in walking speed can be a potential confounder while evaluating STV.
\end{abstract}

\section{Background}

Human walking is an automated rhythmic motor behavior [1-3]. Automaticity and rhythmicity imply that a healthy subject is able to reproduce comparable limb- coordinated movements from stride-to-stride while steady state walking $[2,3]$. Stride-to-stride variability is a measure of the consistency of limb movements [2]. In particular, stride time variability [STV], as calculated out off 
the mean and standard deviation [SD] of stride time and expressed as the coefficient of variation [CoV], is a measure of temporal stride kinematic variability related to the control of the rhythmic stepping mechanism. Low variability values of stride time reflect the automated regular rhythmic feature of gait and are associated with safe gait and are used as a clinical index of gait stability [4-9]. Because walking is one of the most repetitive and "hard wired" human movements, STV is low and usually below $3 \%$ among young healthy adults [9-12].

Changes in gait variability outside of the normal range of gait variability must be studied cautiously as several factors may influence STV. Although the relation between an increased STV and certain neuro-degenerative diseases is well-established [i.e., Parkinson's and Alzheimer's disease], the possible effect of a decrease in walking speed on STV has been rarely examined and is still controversially discussed [13-17]. Previous studies obtained conflicting results, as some failed to find any relationship $[12,13]$ while others reported either a linear or a non-linear relationship [14-17]. Additionally, most of these studies did not take into account the effects of potential confounders that may modify the STV, such as between-subjects variability, the repetition of trials, left-right step asymmetry or the use of motorized treadmills [6,7,12-17]. It is therefore unclear whether an increase in STV is provoked either by the decrease in walking speed, by confounders, or both.

The aim of this study was to determine the extent to which a decrease in self-selected walking speed influenced STV among healthy young adults.

\section{Methods \\ Subjects}

Twenty-nine young adults (15 men and 14 women; mean age $28.3 \pm 6.2$ years; range: $18-39$ years) were recruited after having given their informed consent. The participants reported no physical or mental disorders. They were not taking any medication. The study was approved by the local Ethics Committee and conducted in accordance with the ethical standards set forth in the Declaration of Helsinki (1983).

\section{Tasks and procedure}

Participants were asked to straight walk in non-randomized order and consecutively at $80 \%, 70 \%, 60 \%, 50 \%$, $40 \%, 30 \%, 20 \%$ and $10 \%$ of their preferred walking speed. Self-selected speed was freely chosen following the instructions of evaluator to reduce the preferred walking speed by $10 \%$ percentage. The subjects were instructed that they should reduce their walking speed from their preferred walking speed to $10 \%$ of it. The verbal instructions were standardized: "You will straight walking at your self selected walking speed. You will start at your preferred walking speed and you will decrease your walking speed stage by stage of $10 \%$ until walking to $10 \%$ of your preferred walking speed. You will perform 3 trials for each walking speed condition. Have you understood the instructions? Do you have any question about this test?" All subjects started gait recording with their preferred walking speed. Because the design was based on freely chosen walking speed, the real speed decreasing rate differed from the theoretical rate, and was therefore calculated using the following formula: [(measured selfselected stride velocity/preferred stride velocity) $\times 100]$. The results showed that participants actually walked at $89 \%, 80 \%, 72 \%, 65 \%, 58 \%, 53 \%, 46 \%$ and $39 \%$ of their preferred walking speed. Participants completed 3 trials for each level of decrease in walking speed. Before the test was carried out, a trained evaluator gave standardized verbal instructions regarding the test procedure with a visual demonstration of the walking test. The walking trials were carried out in a well-lit environment, with subjects wearing their own footwear. According to the guidelines for spatio-temporal gait analysis, and in order to ensure that gait parameters were collected while steady state walking, participants started walking at least 2 meters before reaching the electronic walkway and completed their walk at least two meters beyond it [18].

\section{Apparatus}

The GAITRite ${ }^{\circledast}$-System (GAITRite Gold, CIR Systems, PA, USA) is an electronic walkway-integrated, pressure-sensitive electronic surface of $7.32 \times 0.61 \mathrm{~m}$, connected to a portable computer via an interface cable [7]. The carpet includes a series of sensors (a total of 13824 sensors) placed every $1.27 \mathrm{~cm}$ with their centers placed in a $48 \times$ 288 grid and activated by mechanical pressure. The data from the activated sensors is collected by a series of onboard processors and transferred to the computer through a serial port. The data is sampled from the carpet at a frequency of $80 \mathrm{~Hz}$, allowing a temporal resolution of 12.5 ms. Stride time (i.e. gait cycle duration) is defined as the time elapsed between the first contact of two consecutive footsteps of the same foot and is expressed in milliseconds.

\section{Outcomes}

The following outcome variables were used: Mean value and SD expressed in second and $\mathrm{CoV}$ of stride time $(\mathrm{CoV}$ $=[(\mathrm{SD} /$ mean $) \times 100]$ expressed in percentage; and Mean value of stride velocity for each walking condition expressed in $\mathrm{cm} \cdot \mathrm{s}^{-1}$. The primary outcome measure was $\mathrm{CoV}$ of stride time. Mean value and SD of stride time, and Mean value of stride velocity were the secondary outcomes measures. Repetitions of trial, subjects' effect and step asymmetry were used as covariates in data analysis.

\section{Data analysis}

Stride time and stride velocity values were summarized using means and standard deviations. The normality of 
the parameters' distribution was verified with skewness and kurtosis tests before and after applying usual transformations to try to normalize non-Gaussian variables. As these transformations were unable to achieve normalization of the distribution, raw values had to be used. Firstly, a comparison of the outcomes, based on the Kruskal-Wallis test, was carried out for the 3 trials of each walking condition performed. The aim was to convert the three trials into one single trial if no significant difference between trials was observed. The Spearman Brown prophecy coefficient was used to estimates test-retest reliability of the 3 trials. Secondly, a comparison of stride velocity for each level of decrease in walking speed was performed using the Cuzick test. Thirdly, a balanced repeated measures analysis of variance (ANOVA) was performed in order to estimate the effects of a decrease in walking speed on the mean value, $\mathrm{SD}$ and $\mathrm{CV}$ of stride time while adjusting for the 3 trial repetitions, for each walking condition and for subjects' effect, taking account of the variability between subjects and step asymmetry without interactions terms. Fourthly, a univariate quadratic regression was performed to separately explore the association between decreased walking speed and mean value, $\mathrm{SD}$, and $\mathrm{CoV}$ of stride time, respectively; the preferred walking speed served as reference level. $P<0.05$ was considered statistically significant. Our statistics were calculated using the Stata Statistical Software, release 9.2 [19].

\section{Results}

The mean values and SD of stride time and stride velocity parameters are summarized in a Table [See additional file 1]. There was no significant difference for all stride parameters between the 3 trials for each level of decrease in walking speed. The stride velocity decreased significantly from 88 to $39 \%$ of the preferred waking speed (p-trend < 0.001). The ANOVA model (Table 1). showed that the decrease in stride velocity explained the increase in mean values, $\mathrm{SD}$ and $\mathrm{CoV}$ of stride time $(\mathrm{p}<0.001)$, whereas the repetition of trials $(\mathrm{p}=0.534, \mathrm{p}=0.177$ and $\mathrm{p}=0.691$ respectively for mean, $\mathrm{SD}, \mathrm{CoV})$; and step asymmetry $(\mathrm{p}=$ 0.971, $\mathrm{p}=0.150$ and $\mathrm{p}=0.288$ for mean, SD and CoV) had no significant effect. The estimated trial reliability amounted to $96.3 \%$ for the mean, $93.1 \%$ for the SD and $89.9 \%$ for the CV of stride time. Additionally, the subject's effect was significant for all stride parameters $(\mathrm{p}<0.001)$. As shown in figures 1, 2 and 3, the relationship between a decrease in walking speed and stride parameters was quadratic and showed higher STV at a slow speed ( $\mathrm{p}<$ $0.001)$.

\section{Discussion}

Our results show that STV increased while walking speed decreased, even when taking into account an adjustment for the subjects' effect, the repetition of trials and the leftright step asymmetry. This finding has two main implications. Firstly, gait may become more unstable as walking

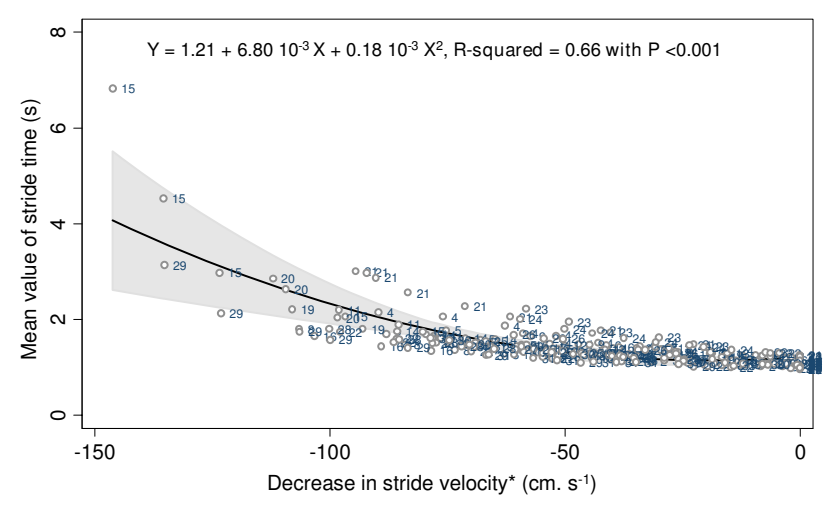

Figure I

Quadratic regression inquiring into a possible association between mean value of stride time and decrease in self-selected walking speed, with the reference value set as the normal self-selected walking speed among young healthy adults $(\mathbf{n}=29)$. *: Normal self-selected walking speed used as the reference level and coded as $0 \mathrm{~cm} \cdot \mathrm{s}^{-1}$.

speed decreases. Secondly, the decrease in walking speed should be considered as a potential confounder while evaluating STV in gait disorders associated with a decrease in walking speed.

The relationship between STV and walking speed is complex and not fully established, as some studies failed to find any relationship $[12,13]$, while others reported either a linear or a non-linear relationship [14-17]. Our study

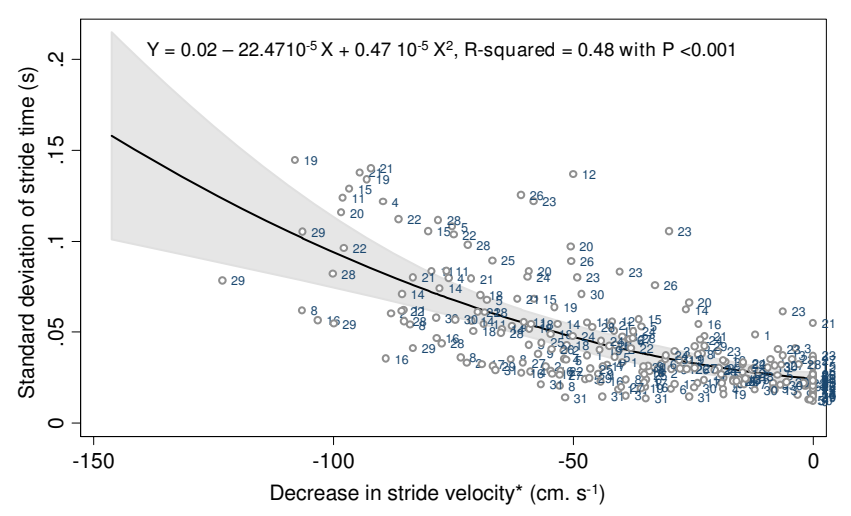

Figure 2

Quadratic regression inquiring into a possible association between standard deviation of stride time and decrease in self-selected walking speed, with the reference value set as the normal self-selected walking speed among young healthy adults $(\mathbf{n}=29)$. *: Normal self-selected walking speed used as the reference level and coded as $0 \mathrm{~cm} \cdot \mathrm{s}^{-1}$. 


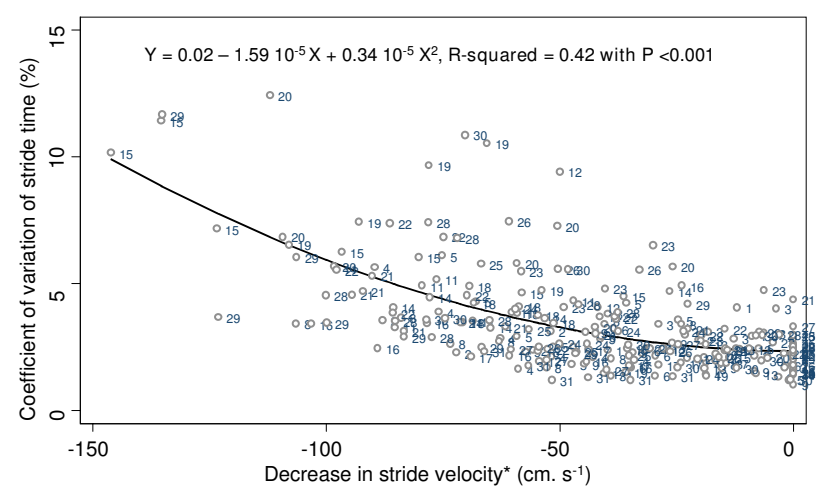

Figure 3

Quadratic regression inquiring into a possible association between coefficient of variation of stride time and decrease in self-selected walking speed, with the reference value set as the normal self-selected walking speed among young healthy adults $(n=29)$. *: Normal self-selected walking speed used as the reference level and coded as $0 \mathrm{~cm} \cdot \mathrm{s}^{-1}$.

solely focused on the effects of decreased walking speed, whereas previous studies analyzed both increased and decreased walking speeds, and corroborated earlier data establishing a significant negative correlation between STV and walking speed [14-17]. Only two studies did not find any relationship between gait speed and stride time variability $[12,13]$. This apparent controversy may result from the fact that only the effect of a low walking speed decrease was examined in these two studies. It has been shown that a higher STV was reported at very slow walking speeds (e.g. 0.2 to $0.6 \mathrm{~m} . \mathrm{s}^{-1}$ ), compared to moderated speeds (e.g. 0.8 to $1.4 \mathrm{~m} . \mathrm{s}^{-1}$ )[16]. Subjects walked at a walking speed $10 \%$ slower than their preferred speed, corresponding to walking at $0.9 \mathrm{~m} . \mathrm{s}^{-1}$ in Owing's study [13] and $1.0 \mathrm{~m} . \mathrm{s}^{-1}$ in Frenkel-Toledo's study [12]. Therefore, the decrease in walking speed was probably too small to show a significant increase in STV. Recently, Jordan et al. [15] also showed a similar negative association between walking speed and STV. STV decreased while walking speed increased. However, unlike in our study design, both decreased and increased walking speeds were used as a basis to report this significant relationship. Lower STV was only observed with fast walking speed and not with preferred speed.

Our results provide the evidence that the relationship between decreased walking speed and increased STV is not linear but quadratic. Heiderscheit [16] was the first to suggest a U-shaped curved non-linear relationship between STV and walking speed, whereby higher STV was observed at slow and fast speed, whereas lower STV was recorded at preferred speed. However, no statistical analysis was performed to confirm these descriptive results. There are several others arguments in favor of a non-linear relationship, provided by the analysis of walk-run and run-walk transition ranges of walking speed. Brisswalter \& Mottet [20] observed a consistent level of variability before and after these transitions. Furthermore, Belli et al. [14] also reported that STV significantly increased while walking speed changed from preferred speed to $140 \%$ at maximal speed. In addition, these results support the assumption that cyclic movements, like limb-movements while walking, have maximal cycle variability at specific cycle frequencies [3].

In contrast to previous studies [12-17], we took into account the role of potential confounders that may modify the relationship between STV and walking speed. Firstly, the subjects' effect was considered as a variable that may in part explain the increased STV. In order to compute the error term in the ANOVA model, the subjects were nested within walking conditions. The subjects' effect is a specific case of group effect, with each group having only one member; when one deals with repeated measures, meaning that many observations are recorded for the same subjects. The subjects' effect allows adjusting

Table I: $P$-value of repeated measures analysis of variance (ANOVA) $(n=\mid 280$ steps) estimating the effects of a decrease in selfselected walking speed on mean value, standard deviation and coefficient of variation of stride time, adjusted for subject's effect $(n=$ 29), number of trials per walking condition and left-right step asymmetry

\begin{tabular}{|c|c|c|c|c|c|}
\hline \multirow[b]{2}{*}{ Stride time } & \multicolumn{4}{|c|}{ Effect } & \multirow[b]{2}{*}{ R-squared } \\
\hline & Decrease in preferred walking speed & Subject & Trials* & Left-right step asymmetry† & \\
\hline Mean value & $<0.001$ & $<0.001$ & 0.534 & 0.971 & 0.612 \\
\hline SD & $<0.001$ & $<0.001$ & 0.177 & 0.150 & 0.481 \\
\hline CoV & $<0.001$ & $<0.001$ & 0.691 & 0.288 & 0.293 \\
\hline
\end{tabular}

SD: standard deviation,

CoV: Coefficient of variation expressed in percentage and calculated from the formula: [(Standard deviation/Mean value) $\times 100]$,

*: Number of trials per walking condition coded in three level $(0=$ first trial, $\mathrm{I}=$ second trial, $2=$ third trial)

$t$ : Right and left step coded as a binary variable $(0=$ Right, $I=$ Left $)$ 
a model for some unknown subject's characteristics and provides information on the heterogeneity among subjects that may artificially increase the STV of a group of subjects. Secondly, the development of a motor skill is possible while walking trials are repeated. Many studies used a motorized treadmill that required participants' training before gait parameters could be measured [1215]. It has been shown that limb movement variability decreases as a function of practice and increment of skills [1-3]. The training phase or the repetition of trials may therefore reduce STV. In our study, although no treadmill was used, this confounder was examined and had no impact on STV. Thirdly, an increase in STV may be explained by left-right step asymmetry in healthy subjects $[13,14]$. This confounder was solely controlled for in the study by Owings \& Grabiners [13], in which an absence of left-right stride asymmetry in healthy adults was reported, similarly to the results we have obtained. Fourthly, our study used a specific procedure in which the decreased walking speed was freely chosen following the instruction to reduce the normal walking speed by a certain percentage. Because STV could be influenced by the imposed walking speed of a motorized treadmill [1-3], we used a GAITRite $^{\circledast}$ system [7]. This device is an electronic carpet which specifically respects the ecological walking condition by recording the freely chosen walking speed. Freely chosen walking speed is primordial when examining STV because it is the only walking speed that takes into account the strategy aiming at maintaining an optimal index of movement consistency in terms of energy costs, attentional demand and efficiency of gait control, leading to a low STV $[5,6,9,20]$.

Both increased STV and slow walking speed have been independently related to gait instability $[4,6,9,11,21]$. Therefore, the main implication of our results is that gait may become more unstable when the walking speed decreases. Instability could be related to a quality change in gait control which becomes less efficient with slower speed compared to preferred speed. STV reflects the control of the walking-related rhythmic stepping mechanism [2], which mainly depends on the basal ganglia and the spinal central pattern generator [1]. Low STV variability reflects the automatic processes associated with an efficient gait control and high gait safety $[2,6]$. An increased STV has been associated with the involvement of higherlevel gait control [22], suggesting that the STV increase shown in our study could be a marker of cortical gait control. However, Dubost et al. [6] showed that the decrease in walking speed was an independent biomechanical factor, significantly related to an increased STV among healthy subjects performing a dual-task. An increase in STV while walking speed decreases among healthy subjects could therefore be solely related to a biomechanical feature of gait, and not necessarily to the involvement of cortical gait control.

STV assessment is a new challenge for clinicians as it provides objective, useful information for the diagnosis of gait instability $[2,4,6,23]$. Moreover, the recently available user-friendly portable gait analysis systems allow a simple, objective STV measurement $[7,8]$, making the assessment of STV possible in clinical practice. However; our results suggest that a decrease in walking speed should be considered as a potential confounder while evaluating STV with the aim to diagnose gait disorders leading to instability. As example, increased STV has been associated with the efficiency of executive function $[24,25]$. In particular, Sheridan et al.. [24], and more recently Allali et al. [25], reported a significant relationship between a high $\mathrm{CoV}$ of stride time and impaired executive function among demented older adults. In both of these studies, it has been suggested that an increase in STV was an index of impaired executive function. However, none of these studies had adjusted the data for a decrease in walking speed. As consequence, we suggest that walking speed should be taken into account while exploring stride time variability in subjects with impaired executive function because increase in stride time variability could be provoked by either impaired executive function or a decrease in walking speed, or by both.

In regard to methodology, a limitation of our study could be related to the number of strides required to obtain a suitable, representative measure of gait variability. When analyzing steady-state walking across $22 \mathrm{~m}(7.32 \mathrm{~m} \times 3$ trials), the number of strides observed was low compared to those recommended by Owings \& Grabiner, who suggested that an accurate estimation of gait variability required at least 400 steps [26]. However, previous studies recorded fewer strides than we did in our study and obtained relevant results for STV $[6,9]$. We therefore believe that our results are reliable as shown with trials reliability above $89 \%$. A second limitation of our study might be linked to the repetition of trials, which may have induced motor skill learning and thus reduced STV $[1,3]$. The third one is the non Gaussian distribution of the variables, but the statistical procedures used are robust enough given the number of subject above 25 and the large number of steps recorded. The fourth limitation is the impossibility to generalize our results because we limited analyze to healthy young adults.

\section{Conclusion}

We have established in the studied sample of young healthy adults a non-linear relation between STV and walking speed, whereby higher STV was reported at a slow speed, supporting the general assumption that gait could 
be more unstable when healthy subjects walk slower than at their normal, i.e. preferred self-selected walking speed. Furthermore, our results highlighted that a decrease in walking speed is a potential confounder while evaluating STV.

\section{Competing interests}

The authors declare that they have no competing interests.

\section{Authors' contributions}

OB has full access to the data in the study and takes responsibility for the integrity of the data and the accuracy of the data analyses. Study concept and design: OB, RWK and YL. Acquisition of data: YL, VD, and CA. Analysis and interpretation of data: OB, GA, FRH, VD, RWK and CA. Drafting of the manuscript: $\mathrm{OB}, \mathrm{GA}, \mathrm{CA}$ and RWK. Critical revision of the manuscript for important intellectual content: CA, FRH, GA and YL. Statistical expertise: FRH. Administrative, technical, or material support: VD and CA. Study supervision: OB and RWK.

All authors have read and approved the final manuscript

\section{Additional material}

\section{Additional file 1}

Mean value and standard deviation of stride time parameters and stride velocity $(n=29)$. The data provided show mean value and standard deviation of stride time parameters and stride velocity $(n=29)$. SD: standard deviation, $\mathrm{CoV}$ : Coefficient of variation expressed in percentage and calculated from the formula: [(Standard deviation/Mean value) $\times$ 100]; *: Mean value of the 3 trials; $t$ : Comparison between the 3 trials for each walking condition, based on Kruskal-Wallis test.

Click here for file

[http://www.biomedcentral.com/content/supplementary/17430003-6-32-S1.doc]

\section{Acknowledgements}

We are grateful to the participants for their cooperation and Raphaël Grandjean for data management.

\section{References}

I. Nutt JG, Marsden CD, Thompson PD: Human walking and higher-level gait disorders, particularly in the elderly. Neurology 1993, 43:268-79.

2. Gabell A, Nayak US: The effect of age on variability in gait. J Gerontol 1984, 39:662-6.

3. Newell KM, Corcos DM: Issues in variability and motor control. In Variability and motor control Edited by: Newell KM, Corcos DM. Champaign: Human Kinetics; 1993: I-I2.

4. Hausdorff JM, Rios DA, Eldelberg HK: Gait variability and fall risk in community-living older adults: a I-year prospective study. Arch Phys Med Rehabil 200 I, 82:1050-6.

5. Beauchet $O$, Berrut G: Gait and dual-task: definition, interest, and perspectives in the elderly. Psychol Neuropsychiatr Vieil 2006, 4:215-25.

6. Dubost V, Kressig RW, Gonthier R, Herrmann FR, Aminian K, Najafi $B$, Beauchet O: Relationships between dual-task related changes in stride velocity and stride time variability in healthy older adults. Hum Mov Sci 2006, 25:372-82.

7. Bilney $B$, Morris $M$, Webster K: Concurrent related validity of the GAITrite ${ }^{\circledR}$ walkway system for quantification of the spatial and temporal parameters of gait. Gait Posture 2003, I 7:68-74.

8. Beauchet O, Herrmann FR, Grandjean R, Dubost V, Allali G: Concurrent validity of SMTEC ${ }^{\circledR}$ footswitches system for the measurement of temporal gait parameters. Gait Posture 2008 , 27:156-9.

9. Beauchet O, Najafi B, Dubost V, Aminian K, Mourey F, Kressig RW: Age-related decline of gait control under a dual-task condition. J Am Geriatr Soc 2003, 5 I : I I 87-8.

10. Goldberger AL, Amaral LA, Hausdorff JM, Ivanov PCh, Peng CK, Stanley HE: Fractal dynamics in physiology: alterations with disease and aging. Proc Natl Acad Sci USA 2002, 99:2466-72.

II. Hausdorff JM, Zemany L, Peng C, Goldberger AL: Maturation of gait dynamics: stride-to-stride variability and its temporal organization in children. J Appl Physiol 1999, 86:1040-7.

12. Frenkel-Toledo S, Giladi N, Peretz C, Herman T, Gruendlinger L, Hausdorff JM: Effect of gait speed on gait rhythmicity in Parkinson's disease: variability of stride time and swing time respond differently. J Neuroengineering Rehabil 2005, 2:23.

13. Owings TM, Grabiner MD: Variability of step kinematics in young and older adults. Gait Posture 2004, 20:26-9.

14. Belli A, Lacour JR, Komi PV, Candau R, Denis C: Mechanical step variability during treadmill running. Eur J Appl Physiol Occup Physiol 1995, 70:510-7.

I5. Jordan K, Challis JH, Newell KM: Walking speed influences on gait cycle variability. Gait Posture 2007, 26: I28-34.

16. Heiderscheit BC: Movement variability as a clinical measure for locomotion. J Appl Biomech 2000, I 6:4 I9-27.

17. Maruyama $H$, Nagasaki $H$ : Temporal varaibility in the phase durations during treadmill walking. Hum Mov Sci 1992, I I:335-48.

18. Kressig RW, Beauchet O, European GAITRite Network Group: Guidelines for clinical applications of spatio-temporal gait analysis in older adults. Aging Clin Exp Res 2006, I 8: I 74-6.

19. Stata Statistical Software: Release 9.2 [program]. College Station, Texas, USA: Stata Corporation; 2003.

20. Brisswalter J, Mottet D: Energy cost and stride duration variability at preferred transition gait speed between walking and running. Can J Appl Physiol 1996, 2 I:47I-80.

21. Snijders AH, Warrenburg BP van de, Giladi N, Bloem BR: Neurological gait disorders in elderly people: clinical approach and classification. Lancet Neurol 2007, 6:63-74.

22. Beauchet O, Dubost V, Aminian K, Gonthier R, Kressig RW: Dualtask-related gait changes in the elderly: does the type of cognitive task matter? J Mot Behav 2005, 37:259-64.

23. Beauchet $O$, Allali G, Berrut G, Dubost V: Is low lower-limb kinematic variability always an index of stability? Gait Posture 2007, 26:327-8.

24. Sheridan PL, Solomont J, Kowall N, Hausdorff JM: Influence of executive function on locomotor function: divided attention increases gait variability in Alzheimer's disease. J Am Geriatr Soc 2003, 51:1633-7.

25. Allali G, Kressig RW, Assal F, Herrmann FR, Dubost V, Beauchet O: Changes in gait while backward counting in demented older adults with frontal lobe dysfunction. Gait Posture 2007, 26:572-6.

26. Owings TM, Grabiner MD: Measuring step kinematic variability on an instrumented treadmill: how many steps are enough? J Biomech 2003, 36:1215-8. 